\title{
Sesión de hemodiálisis en pacientes con peso inferior a 6 kilos
}

\section{Ma Ángeles Coyo Montero - Begoña Díez Polo - Paloma Conde García - Roser Tosquella Amblás}

\section{Hospital Universitario Materno-infantil Vall d'Hebron. Barcelona}

\section{Introducción}

En este caso describimos la práctica de hemodiálisis en un niño de $4 \mathrm{Kg}$. Este procedimiento se practica en nuestro hospital cuando no es posible la diálisis peritoneal y supone el empleo de un equipo técnico específico por parte de un personal experto entrenado en su manejo. Las características del paciente hacen que la prevención y tratamiento de complicaciones inherentes a la hemodiálisis como los trastornos hemodinámicos, la hipotensión, la coagulación de circuito o el mantenimiento y cuidados del acceso vascular, cobren aquí especial relevancia.

\section{Resumen historia clínica}

Recién nacido pretermino (32 semanas). Apgar 8/9 con peso $2350 \mathrm{gr}$. Ecografías prenatales en la semana 32: quistes renales, megaureteres y oligoamnios severos e hipoplasia pulmonar. Precisa ventilación mecánica durante 26 días.

Diagnosticado al mes de vida de insuficiencia renal crónica secundaria a uropatía (síndrome de Prumebelly), reflujo vesicoureteral grado IV en riñón izquierdo y dilatación de uretra posterior con obstrucción severa.

\begin{tabular}{|c|}
\hline $\begin{array}{c}\text { Correspondencia: } \\
M^{a} \text { Ángeles Coyo Montero } \\
\text { Servicio Nefrología-Transplante renal } \\
\text { Hospital Universitario Materno-infantil Vall d'Hebron } \\
\text { Pg. Vall d'Hebron, 129-139 } \\
\text { 08039 Barcelona } \\
\text { macoyo@vhebron.net }\end{array}$ \\
\hline
\end{tabular}

A los 3 meses de vida se produce situación funcional de insuficiencia renal crónica terminal con mal manejo de líquidos, dos episodios de edema agudo pulmonar en contexto de crisis hipertensiva e hipervolemia, precisando ingreso en la unidad de cuidados intensivos pediátrica. Se coloca un catéter de hemodiálisis tipo Perm-cath $\circledast$ tunelizado en vena yugular interna iniciando sesiones de 3-4 horas de duración tres veces por semana. El niño pesa 4 kilos y presenta valores prediálisis de urea de $160 \mathrm{mg} / \mathrm{dl}$ y de creatinina de $4,5 \mathrm{mg} / \mathrm{dl}$.

Portador de ureterostomia percutanea bilateral debido a hidronefrosis bilateral. A los 8 meses de vida se observa miocardiopatía secundaria a HTA con soplo sistólico II/VI y cardiomegalía. A los 11 meses, nefrectomía bilateral debida a HTA grave con episodios convulsivos de difícil manejo.

La hemodiálisis nos ha permitido que el paciente alcance 6 kilos de peso haciéndolo mucho más asequible para el transplante renal. Se realizó transplante renal en junio del 2007 con buena evolución del injerto, función renal satisfactoria y control de HTA sin medicación.

\section{Problemas a destacar durante el proceso de la hemodiálisis}

1. Trastorno hemodinámico del paciente como consecuencia del volumen circulante del circuito que oscila entre $\pm 30 \mathrm{~cm}^{3}$. Como medida preventiva en las primeras sesiones se cebó el circuito con sangre.

2. Hipotensión como consecuencia de la ultrafiltración a que están sometidos estos pacientes que puede alcanzar los 300-500 ml (5-10\% de su pe- 
so corporal) por el aumento de la cantidad de líquido acumulado interdiálisis debido a la ingesta alimentaría y la anuria. Se prefundió seroalbúmina al $20 \%$ en pequeñas cantidades $( \pm 10 \mathrm{~cm}$ en 1h) manteniendo tensiones diastólicas $5 \mathrm{~mm} \mathrm{Hg}$.

3. Coagulación del circuito y acceso vascular propiciada por la velocidad de los flujos sanguíneos bajos ( $\pm 50-100 \mathrm{ml}$ por minuto) y sesiones de $\pm 3 \mathrm{~h}$. La heparinización se realizó con heparina de bajo peso molecular (2,5-5 mg al inicio, en dosis única).

\begin{tabular}{l|l}
\hline Edad: 4 meses & Peso seco: $3600 \mathrm{gr}$ \\
\hline Talla: $62 \mathrm{~cm}$ & Superficie corporal: 0,3 m2 \\
\hline Inicio de la diálisis: 10-07-2006 & $\begin{array}{l}\text { Baño de bicarbonato: } \\
35 \mathrm{meq} / \mathrm{l}\end{array}$ \\
\hline $\begin{array}{l}\text { Duración de diálisis: } 2 \text { horas } \\
\text { (3 veces/semana) }\end{array}$ & $\begin{array}{l}\text { Tipo de solución: Dialsol 203 } \\
\text { acido/base (Na 140meq/l }\end{array}$ \\
\hline Dializador: FX 2 & yicNa 35meq/l) \\
\hline $\begin{array}{l}\text { Flujo de sangre (Qb): } \\
\text { 50-100 ml/min. }\end{array}$ & \begin{tabular}{l} 
(modelo 4008) \\
\hline Temperatura baño diálisis: $37^{\circ} \mathrm{C}$
\end{tabular} \\
\hline Medicación en diálisis: Clexane $2,5 \mathrm{mg}$ al inicio, Eprex 500 ui \\
post hemodiálisis
\end{tabular}

Esquema de la hemodiálisis

\section{Actuaciones de Enfermería}

\section{Llegada del niño a la unidad}

Recibir al paciente valorando:

- Estado general: descanso, sueño, comodidad, signos de dolor.

- Higiene: aseo, estado de los apósitos del acceso vascular.

- Alimentación: necesidades calóricas, ultima toma administrada, estado nutricional.

- Fármacos: medicación prescrita, control del calendario vacunal, etc

\section{Actuaciones antes de la conexión}

- Pesar al niño: en pesabebés y completamente desnudo.
- Colocar al niño en la cama: vestido con camiseta y pañal.

- Realizar una sujeción cómoda y segura

- Mantener ambiente que favorezca el descanso.

- Realizar monitorización en situaciones críticas 0 primeras sesiones.

- Medición de constantes según necesidades: TA, FC, FR y temperatura.

- Cuidados del acceso vascular:

- Asepsia rigurosa antes de manipular el catéter.

- Comprobar zona de inserción.

- Control de signos de infección: inflamación, tumefacción u otros signos en punto de inserción y alrededores.

- Comprobar y mantener permeabilidad del catéter: sacar coágulo por las dos luces con la mínima pérdida hemática.

\section{Actuaciones en la conexión}

- Cálculo e introducción de los datos dialíticos en el monitor

- Extracción de analítica prediálisis: hemograma, bioquímica, gases, coagulación etc. según precise el niño para evitar punciones fuera de la sesión.

- Conexión al monitor de HD con la máxima asepsia.

- Administración de heparina de bajo peso molecular en dosis única según peso seco.

- Sujeción segura de las líneas.

- Fijación del acceso vascular evitando manipulaciones del niño, respetando la máxima movilidad.

- Tener especial atención con los movimientos del niño.

- Administración de la toma alimenticia que le corresponde.

- Control de constantes según necesidades: en la monitorización y cada 10 minutos en los registros.

\section{Actuaciones en la desconexión}

- Asepsia y colocación de un campo. Retorno del circuito con solución glucosada al 5\% ( $\pm 20 \mathrm{cc})$ 
- Administración de medicaciones: vía endovenosa, antibióticos, etc.

- Realizar control analítico si es necesario post hemodiálisis

- Heparinizar el catéter con heparina sódica diluida con solución salina. Se infunde el volumen interno del catéter especificado en cada luz.

- Fijación cómoda y segura del acceso vascular con dispositivo de fijación de catéter).

- Apósito de fijación de tipo Statlock o similar.

- Observación del estado general y de constantes vitales

- Control de peso seco post diálisis para balance.

- Anotación de todos los datos en hoja de registro de enfermería.

\section{Aspectos técnicos de la HD}

- Catéter:

- Tipo Perm-cath® tunelizado

- Tamaño 8 FR x $18 \mathrm{~cm}$

- Localización en vena yugular interna

- Dializador

- Fx paed $2 \circledR$

- Superficie de 0,2 m2

- Volumen cebado de 18 cc

- Líneas

- Tipo neonatales

- Segmento de bomba 4,4 mm de grosor

- Volumen de cebado de 22 cc

- Monitor

- Que permita modificar parámetros y se adapte a pediatría

- Segmento de bomba adaptado a líneas neonatales

\section{Conclusiones}

Siempre que no sea posible realizar una diálisis peritoneal, la aparición de nuevos equipos y material adecuado (líneas neonatales y dializador pequeño), para dializar a pacientes con un peso inferior a $6 \mathrm{ki}$ los, ha permitido que, en nuestra unidad, podamos llevar a cabo la hemodiálisis con buenos resultados, en pacientes agudos o crónicos con bajo peso, hasta que alcancen el peso y tamaño que puedan facilitar un mejor resultado en el transplante renal.

No obstante, tanto el bajo peso como la gran labilidad de estos pacientes hacen que la práctica de la hemodiálisis requiera de equipo técnico específico y que la técnica se realice por parte de personal experto y entrenado en su manejo.

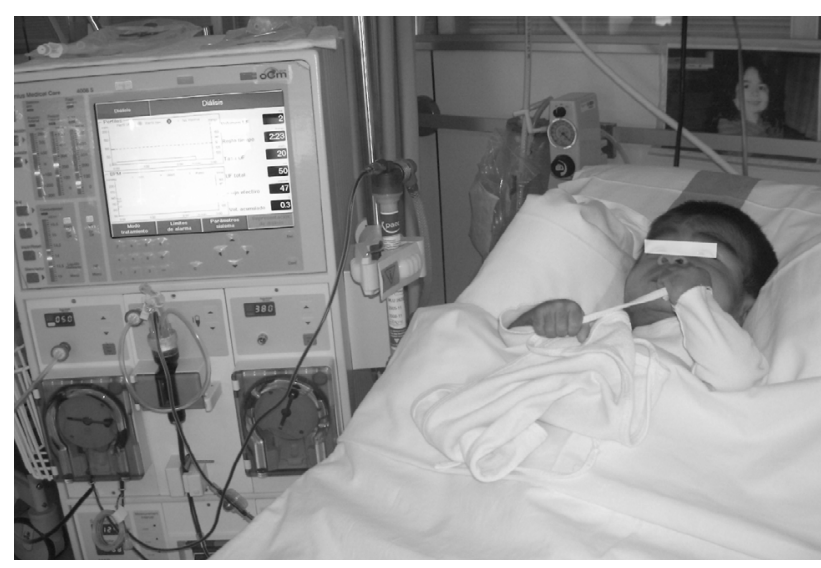

Figura 1. El niño está tranquilo durante la sesión de hemodiálisis. En la imagen se puede apreciar el dializador pequeño y las líneas neonatales.

\section{Bibliografía}

1. Shoroff RW, Ledereman S, Hutchinson C and Rees L. Chronic hemodiálisis in infants and children under 2 years of age. Pediatr Nephrol 2003; 18:378-383.

2. Neu Am, Ho PL, McDonald RA and Warady BA. Chronic diálisis in children and adolecents. The 2001 NAPRATCS Annual Report. Pediatr Nephrol 2002; 17:656-663.

3. Leonard Ma, Donaldson IA, HO m and Geay FA. A prospective cohort study of incident maintenace diálisis in children: An NAPRTCS study. Kideney Int 2003; 63: 744-755.

4. Patel NH, Revanur VK, Khanna A, Hodges $C$ and Jindal RM: Vascular acces for hemodiálisis: an indepth review. J Nephrol 2001; 14:146-156.

5. Sheth RD, Brandt MI, Brewer EF, Nuchenter JG, Kale AS and Goldstein SL: Permanent hemodiálisis vascular acces survival in children and adoescents with end stage renal disease. Kidney Int 2005; 62:1864-1869 